\title{
Association of changes of pulse wave velocity and augmentation index after isometric handgrip exercise with coronary lesion extent and revascularization
}

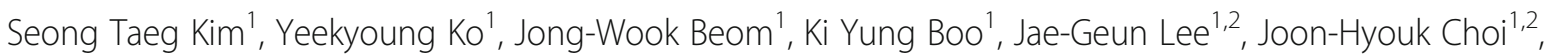
Song-Yi Kim ${ }^{1,2}$ and Seung-Jae Joo $1,2,3^{*}$ (iD

\begin{abstract}
Background: Arterial stiffness is associated with myocardial ischemia and incident coronary artery disease (CAD), and indexes of arterial stiffness are usually increased in patients with CAD. However, these indexes are often increased in elderly without CAD. Arterial stiffness in patients with CAD may become more evident after isometric handgrip exercise which increases systolic pressure and ventricular afterload. We investigated the association of the change of stiffness indexes after isometric handgrip exercise with the lesion extent of CAD and the necessity for coronary revascularization.
\end{abstract}

Methods: Patients who were scheduled a routine coronary angiography via a femoral artery were enrolled. Arterial waveforms were traced at aortic root and external iliac artery using coronary catheters at baseline and 3 min after handgrip exercise. Augmentation index (AIx) was measured on the recorded aortic pressure waveform, and pulse wave velocity (PWV) was calculated using the ECG-gated time difference of the upstroke of arterial waveforms and distance between aortic root and external iliac artery.

Results: Total 37 patients were evaluated. Both PWV and Alx increased after handgrip exercise. $\triangle$ PWV was significantly correlated with $\Delta \mathrm{Alx}(r=0.344, P=0.037)$. Patients were divided into higher and lower $\Delta \mathrm{PWV}$ or $\Delta \mathrm{Alx}$ groups based on the median values of $0.4 \mathrm{~m} / \mathrm{sec}$ and $3.3 \%$, respectively. Patients with higher PWV had more 2- or 3-vessel CAD (69\% vs. 27\%, $P=0.034)$, and underwent percutaneous coronary intervention $(\mathrm{PCI})$ more frequently ( $84 \%$ vs. $50 \%, P=0.038$ ), but higher $\triangle A l x$ was not associated with either the lesion extent or $\mathrm{PCl}$. Area under curve (AUC) of $\triangle \mathrm{PWV}$ in association with $\mathrm{PCl}$ by $\mathrm{C}$-statistics was 0.70 (95\% confidence interval $[\mathrm{Cl}] 0.51-0.88 ; P=0.056)$. In multiple logistic regression analysis, $\triangle \mathrm{PWV}$ was significantly associated with $\mathrm{PCl}$ (odds ratio $7.78 ; 95 \% \mathrm{Cl} 1.26-48.02$; $P=0.027)$.

\footnotetext{
* Correspondence: sejjoo@jejunu.ac.kr

'Department of Internal Medicine, Jeju National University Hospital, 15 Aran

13-gil, Jeju City, Jeju Province 63241, Republic of Korea

${ }^{2}$ Department of Internal Medicine, Jeju National University School of Medicine, 15 Aran 13-gil, Jeju City, Jeju Province 63241, Republic of Korea Full list of author information is available at the end of the article
}

(c) The Author(s). 2021 Open Access This article is licensed under a Creative Commons Attribution 4.0 International License, which permits use, sharing, adaptation, distribution and reproduction in any medium or format, as long as you give appropriate credit to the original author(s) and the source, provide a link to the Creative Commons licence, and indicate if changes were made. The images or other third party material in this article are included in the article's Creative Commons licence, unless indicated otherwise in a credit line to the material. If material is not included in the article's Creative Commons licence and your intended use is not permitted by statutory regulation or exceeds the permitted use, you will need to obtain permission directly from the copyright holder. To view a copy of this licence, visit http://creativecommons.org/licenses/by/4.0/. The Creative Commons Public Domain Dedication waiver (http://creativecommons.org/publicdomain/zero/1.0/) applies to the data made available in this article, unless otherwise stated in a credit line to the data. 
(Continued from previous page)

Conclusions: Higher $\triangle \mathrm{PW}$ after isometric handgrip exercise was associated with the lesion extent of CAD and the necessity for coronary revascularization, but higher $\Delta$ Alx was not.

Keywords: Arterial stiffness, Pulse wave velocity, Pulse wave analysis, Isometric exercise, Percutaneous coronary intervention

\section{Background}

Normal or accelerated vascular aging and hypertension are two main factors determining arterial stiffness because they are basically related to the change of the arterial media [1]. However, diabetes, dyslipidemia, and smoking in addition to vascular aging and hypertension, all of which are shared common risk factors for coronary artery disease (CAD), may also change the mechanical properties of the arterial wall to make a stiff artery [2]. In a stiff arterial system, the velocities of the incident flow and the backward reflection flow are rapid enough for the early return of reflected waves during arterial systole rather than diastole, and the incident and reflected pressure waves are summed to increase aortic or central systolic pressure, but decrease central diastolic pressure, resulting in widened central pulse pressure [3]. Augmented central systolic pressure increases ventricular afterload and myocardial oxygen demand, and lowered diastolic pressure decreases coronary blood perfusion: the net results are myocardial ischemia and ventricular dysfunction [3-6]. For this reason, a stiff arterial system is associated with incident CAD, heart failure or stroke [7-9], and indexes of stiffness are related to the presence and severity of CAD [5]. Therefore, a stiff large artery is another feature of patients with CAD.

One of the popular methods evaluating arterial stiffness is measuring pulse wave velocity (PWV), a speed of an arterial pulsation through the arterial tree, usually between carotid and femoral arteries [6]. A number of studies reported that PWV was increased in patients with $\mathrm{CAD}$, and correlated with the severity of coronary atherosclerosis [5, 10-14]. Increased aortic PWV (>10 $\mathrm{m} / \mathrm{sec}$ ) is considered as a marker for detecting hypertension-mediated organ damage in guidelines for the management of arterial hypertension $[15,16]$. Another method for measuring the arterial stiffness is central pulse wave analysis. Augmentation by the summation of incident and reflected waves in the aortic pressure waveform is expressed as augmentation pressure (AP) or augmentation index (AIx). AIx is a percentage of AP on aortic pulse pressure $[3,6]$. The earlier studies showed that AIx was increased in patients with $\mathrm{CAD}$ and was associated with the severity of CAD, especially in younger patients $(<60$ years of age) $[10,17,18]$. We already reported that AIx was negatively correlated with minimal luminal area of coronary atherosclerosis measured by intravascular ultrasound, and was associated with coronary revascularization [19]. However, other studies showed no association of AIx with CAD, especially in elderly patients $[8,12,20]$.

Increased PWV or AIx in patients with CAD may become more evident with a maneuver that influences the velocities of incident and reflected pressure waves. Isometric handgrip exercise, which increases systolic blood pressure (BP) and ventricular afterload [21-23], is a suitable and easily applicable tool for this purpose. The changes of PWV and AIx after isometric handgrip exercise may unmask the lesion extent and the necessity for coronary revascularization in elderly patients with CAD. In this study, the association of the change of PWV or AIx after isometric handgrip exercise with the lesion extent of CAD and the necessity for coronary revascularization was investigated.

\section{Methods \\ Study patients}

Patients who were scheduled a routine coronary angiography (CAG) via a femoral artery for the evaluation of the coronary atherosclerosis, and agreed to participate in the study were enrolled. Patients with acute coronary syndrome, or valvular heart disease were excluded. CAD was defined as $\geq 70 \%$ stenosis of the luminal diameter by the visual estimation of the operator in at least one major epicardial coronary artery or a past medical history of coronary revascularization. The extent of coronary atherosclerosis was classified as 1-, 2- or 3-vessel disease by the number of the major epicardial coronary arteries with a significant stenosis. Patients with a significant stenosis in the left main coronary artery were counted as having 2-vessel disease.

The study protocol was approved by the institutional review board of Jeju National University Hospital. Written informed consents were obtained from participating patients or legal representative.

\section{Study protocol}

The maximal voluntary forearm contraction power was measured with a JAMAR dynamometer (Sammons Preston Rolyan, Nottinghamshire, UK), and a submaximal target at $30 \sim 40 \%$ of maximal handgrip power was used for 3-min isometric handgrip exercise. 
After routine CAG, arterial pressure waveforms were traced using a right coronary catheter and a fluid-filled pressure transducer system. Central arterial waveforms were recorded at the aortic root and traced before and at 1, 2 and $3 \mathrm{~min}$ after handgrip exercise. Forward pressure was measured at a merging point of the forward and the reflected waves on the recorded aortic pressure waveform. Augmentation pressure was defined as maximal central systolic pressure minus forward pressure. AIx was defined as augmentation pressure divided by central pulse pressure and expressed as a percentage (Fig. 1). Peripheral arterial waveforms were recorded at the external iliac artery before and after 3-min handgrip exercise. The ECG-gated time difference ( $\Delta$ Time) of the upstroke of the central and peripheral arterial waveforms was measured. The distance (D) between the aortic root and the external iliac artery was determined by a tape measure of the catheter length from the tip to the entry point at an arterial sheath minus the length of an arterial sheath $(12 \mathrm{~cm})$. PWV was defined as D divided by $\Delta$ Time (Fig. 1) [24].

\section{Statistics}

Data were expressed as mean \pm standard deviation for continuous variables, and as number (percentage) for categorical variables. Changes of hemodynamic parameters after handgrip exercise were analyzed using paired t-test, or where appropriate, Wilcoxon signed rank test. The association between changes of PWV ( $\triangle \mathrm{PWV})$ and AIx ( $\triangle$ AIx) after handgrip exercise was evaluated using correlation analysis. Patients were divided into higher and lower $\triangle \mathrm{PWV}$ or $\triangle \mathrm{AIx}$ groups based on the each median value. Data between groups were compared using unpaired t-test for continuous variables, and chisquare test for categorical variables. The association between higher $\triangle \mathrm{PWV}$ or $\triangle \mathrm{AIx}$ and coronary revascularization was evaluated using $\mathrm{C}$-statistics and multiple logistic regression analysis after adjusting for age, gender, height, body mass index (BMI), hypertension, diabetes mellitus (DM) and chronic kidney disease (CKD). CKD was defined as estimated glomerular filtration rate $<60 \mathrm{~mL} / \mathrm{min} / 1.73 \mathrm{~m}^{2}$ by Modification of Diet in Renal Disease equation.
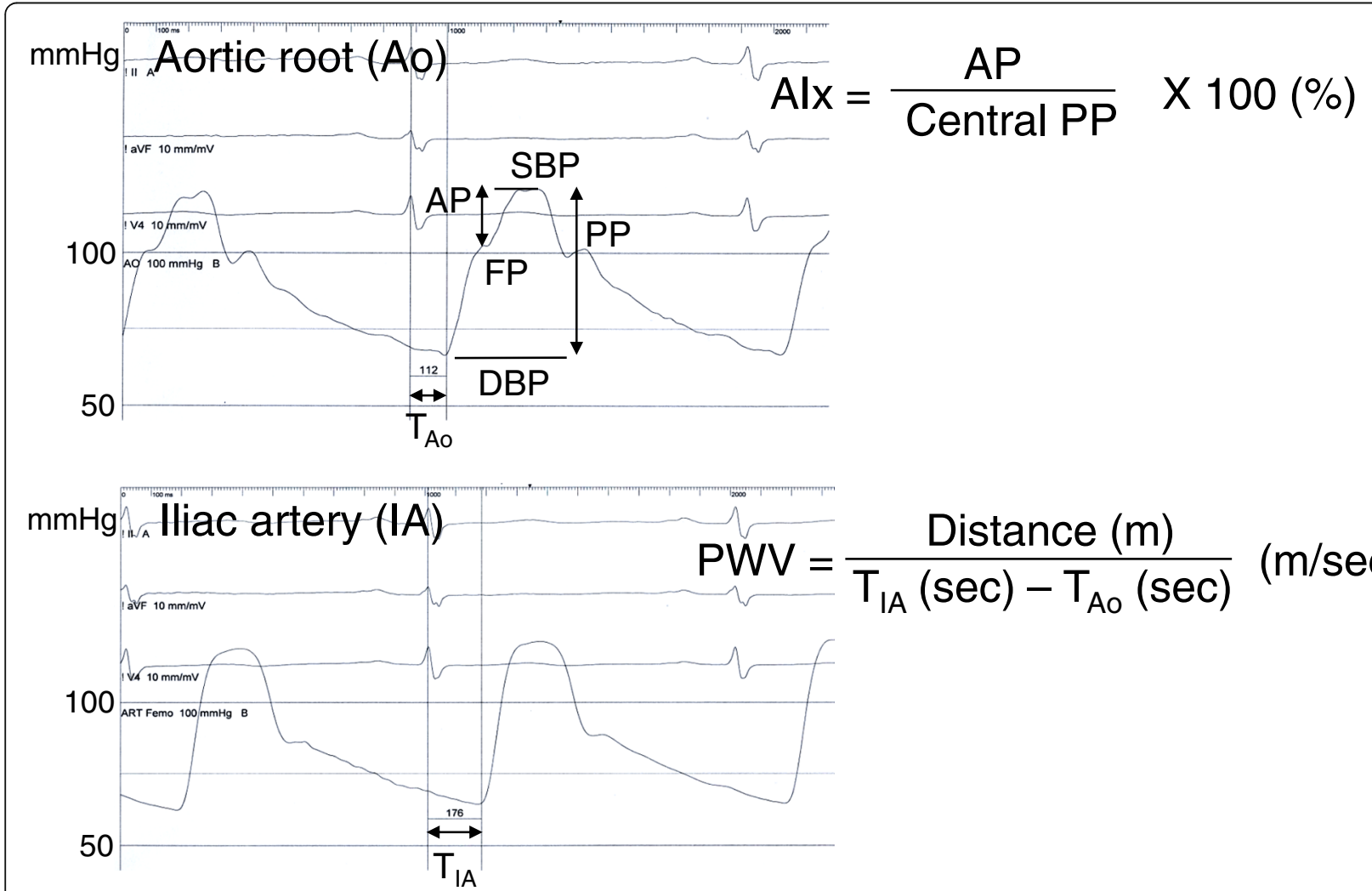

$(\mathrm{m} / \mathrm{sec})$

Fig. 1 Measurements of augmentation index (Alx), and pulse wave velocity (PWV). Forward pressure (FP) was measured at a merging point of the forward and the reflected waves on the recorded aortic pressure waveform. Alx was defined as augmentation pressure (AP) divided by central pulse pressure (PP), and expressed as a percentage. PWV was calculated using the ECG-gated time difference of the upstroke of the arterial waveforms between the aortic root $\left(T_{A O}\right)$ and the external iliac artery $\left(T_{I A}\right)$, and the distance between them determined by a tape measure of the catheter length after removal of a coronary catheter. DBP; diastolic blood pressure, SBP; systolic blood pressure 
Table 1 Changes of hemodynamic parameters after isometric handgrip exercise $(N=37)$

\begin{tabular}{|c|c|c|c|c|}
\hline & Baseline & $1 \mathrm{~min}$ & $2 \mathrm{~min}$ & $3 \min$ \\
\hline Heart rate (/min) & $64.2 \pm 9.2$ & $67.5 \pm 10.1^{*}$ & $68.9 \pm 10.2^{*} \dagger$ & $70.6 \pm 12.9^{*}+$ \\
\hline Central systolic BP (mmHg) & $118.4 \pm 20.6$ & $135.1 \pm 21.4^{*}$ & $138.9 \pm 22.5^{*} \dagger$ & $140.4 \pm 22.9^{*} \dagger$ \\
\hline Central diastolic BP (mmHg) & $63.4 \pm 10.3$ & $71.0 \pm 10.6^{*}$ & $72.1 \pm 11.3^{*}+$ & $72.7 \pm 11.2^{*}+$ \\
\hline Central PP (mmHg) & $55.0 \pm 17.6$ & $64.2 \pm 18.2^{*}$ & $66.8 \pm 19.2^{*} \dagger$ & $67.7 \pm 18.7^{*} \dagger$ \\
\hline Forward pressure (mmHg) & $108.7 \pm 19.4$ & $121.4 \pm 20.7^{*}$ & $124.7 \pm 21.8^{*} \dagger$ & $126.2 \pm 22.6^{*}+$ \\
\hline Augmentation pressure $(\mathrm{mmHg})$ & $9.7 \pm 6.6$ & $13.7 \pm 8.3^{*}$ & $14.2 \pm 8.5^{*}$ & $14.2 \pm 8.7^{*}$ \\
\hline Augmentation index (\%) & $17.4 \pm 10.5$ & $21.2 \pm 11.5^{*}$ & $21.5 \pm 11.5^{*}$ & $21.4 \pm 11.7^{*}$ \\
\hline Peripheral systolic BP (mmHg) & $125.0 \pm 20.4$ & & & $140.7 \pm 25.0^{*}$ \\
\hline Peripheral diastolic BP (mmHg) & $62.2 \pm 10.1$ & & & $65.1 \pm 10.3^{*}$ \\
\hline Peripheral PP (mmHg) & $62.8 \pm 18.4$ & & & $75.7 \pm 21.4^{*}$ \\
\hline Pulse wave velocity (m/sec) & $9.99 \pm 1.84$ & & & $10.81 \pm 2.33^{*}$ \\
\hline
\end{tabular}

Values are mean \pm standard deviation

${ }^{*} p<0.05$ vs. baseline, $t p<0.05$ vs. $1 \mathrm{~min}$

$B P$ Blood pressure, $P P$ Pulse pressure

All statistical analyses were performed with the statistical package SPSS version 23 (IBM Co, Armonk, NY, US). Clinical significance was defined as $P<0.05$.

\section{Results}

Total 42 patients were enrolled. After excluding 4 patients without a definite inflection point of augmentation pressure on the central arterial waveform and 1 patient without a peripheral arterial waveform, final 37 patients were evaluated. The mean age of study patients was $63.3 \pm 9.4$ years (range $41-82$ years), 24 patients $(65 \%)$ were older than 60 years, 26 patients $(70 \%)$ had hypertension, and 27 patients (73\%) had CAD. Percutaneous coronary intervention (PCI) was conducted in 25 patients (68\%): 1 year ago in 20 patients and on the day of the study in 5 patients. One patient underwent both PCI and coronary artery bypass graft.

Heart rate, central systolic and diastolic BP, central pulse pressure, forward pressure, augmentation pressure, and AIx increased progressively from $1 \mathrm{~min}$ after handgrip exercise, and reached the maximal level at $2 \mathrm{~min}$. Peripheral systolic BP, diastolic BP, pulse pressure and

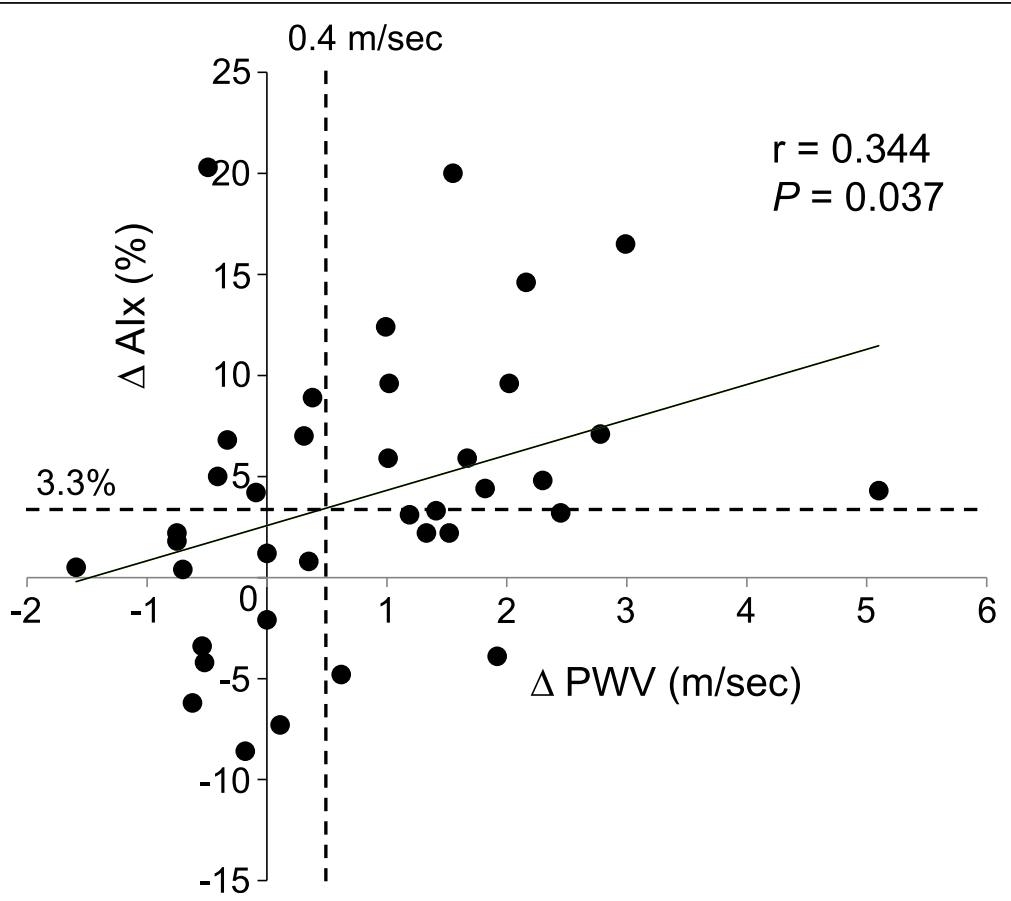

Fig. 2 Correlation between changes of pulse wave velocity $(\Delta \mathrm{PWV})$ and augmentation index $(\Delta \mathrm{Alx})$ after isometric handgrip exercise. The median values of $\triangle \mathrm{PWV}$ and $\Delta \mathrm{Alx}$ were $0.4 \mathrm{~m} / \mathrm{sec}$ and $3.3 \%$, respectively 
PWV also increased after 3-min handgrip exercise (Table 1). $\triangle \mathrm{PWV}$ was significantly correlated with $\triangle \mathrm{AIx}$ $(r=0.344, P=0.037)$ (Fig. 2). The median values of $\triangle \mathrm{PWV}$ and $\Delta \mathrm{AIx}$ were $0.4 \mathrm{~m} / \mathrm{sec}$ and $3.3 \%$, respectively.

Patients were divided into higher and lower $\triangle \mathrm{PWV}$ $(\geq 0.4$ vs. $<0.4 \mathrm{~m} / \mathrm{sec}$ ) groups. Age, gender, BMI, smoker, co-morbid condition such as hypertension, DM, CKD or prior myocardial infarction, and medications were not significantly different between higher and lower $\triangle \mathrm{PWV}$ groups except hyperlipidemia. Patients with higher PWV had more 2 - or 3 -vessel CAD (69\% vs. $27 \%, P=0.034$ ). PCI was more frequently performed in patients with higher PWV ( $84 \%$ vs. $50 \%, P=0.038)$ (Table 2). Baseline hemodynamic parameters including PWV and AIx were not significantly different between two groups. After 3min handgrip exercise, PWV, $\Delta$ central systolic BP, $\Delta \mathrm{AIx}$, and $\Delta$ peripheral systolic BP were greater in patients with higher $\triangle \mathrm{PWV}$ (Table 3).

Patients were also divided into higher and lower $\triangle \mathrm{AIx}$ $(\geq 3.3$ vs. $<3.3 \%)$ groups. Age, BMI, smoker, co-morbid condition such as hypertension, DM, hyperlipidemia,
CKD, prior myocardial infarction, or CAD, and medications except gender were not significantly different between higher and low $\Delta$ AIx groups (Table 4). Patients with 2- or 3-vessel CAD were not significantly different (60\% vs. $42 \%, P=0.313$ ), and PCI was similarly performed in both groups (68\% vs. $67 \%, P=1.000)$. Baseline central systolic BP and pulse pressure, augmentation pressure, AIx, peripheral systolic BP, and PWV were lower in higher $\triangle \mathrm{AIx}$ group. However, after 3-min handgrip exercise, these hemodynamic parameters became not significantly different between both groups (Table 5).

C-statistics showed the area under curve (AUC) of $\triangle \mathrm{PWV}$ in association with PCI was 0.70 (95\% confidence interval $[\mathrm{CI}] 0.51-0.88 ; P=0.056)$. In contrast, it was $0.54(95 \% \mathrm{CI} 0.34-0.75 ; P=0.685)$ in case of $\triangle \mathrm{AIx}$ (Fig. 3). In multiple logistic regression analysis after adjustment, $\triangle \mathrm{PWV}$ was significantly associated with PCI (odds ratio [OR] 7.78; 95\% CI 1.26-48.02; $P=0.027)$, but $\triangle$ AIx was not (OR $2.81 ; 95 \%$ CI $0.37-$ 21.30; $P=0.318$ ).

Table 2 Comparison of baseline characteristics according to the changes of pulse wave velocity after isometric handgrip exercise

\begin{tabular}{|c|c|c|c|c|}
\hline & $\begin{array}{l}\text { All patients } \\
(N=37)\end{array}$ & $\begin{array}{l}\Delta P W V \geq 0.4 \mathrm{~m} / \mathrm{sec} \\
(N=19)\end{array}$ & $\begin{array}{l}\Delta \mathrm{PWV}<0.4 \mathrm{~m} / \mathrm{sec} \\
(N=18)\end{array}$ & $P$ value \\
\hline Age (years) & $63.3 \pm 9.4$ & $63.7 \pm 8.4$ & $62.9 \pm 10.5$ & 0.800 \\
\hline Male & $23(62)$ & $14(74)$ & $9(50)$ & 0.184 \\
\hline Height (cm) & $160.8 \pm 8.9$ & $162.0 \pm 8.3$ & $160.0 \pm 9.6$ & 0.422 \\
\hline Weight (kg) & $65.7 \pm 12.4$ & $66.5 \pm 12.1$ & $64.9 \pm 13.0$ & 0.703 \\
\hline Body mass index $\left(\mathrm{kg} / \mathrm{m}^{2}\right)$ & $25.27 \pm 3.30$ & $25.2 \pm 3.0$ & $25.3 \pm 3.7$ & 0.902 \\
\hline Smoker & $9(24)$ & $5(26)$ & $4(22)$ & 0.459 \\
\hline Hypertension & $26(70)$ & $14(74)$ & $12(67)$ & 0.728 \\
\hline Diabetes mellitus & $8(22)$ & $5(26)$ & $3(17)$ & 0.693 \\
\hline Hyperlipidemia & $33(89)$ & $19(100)$ & $14(78)$ & 0.046 \\
\hline Chronic kidney disease & $19(51)$ & $9(47)$ & $10(56)$ & 0.746 \\
\hline Prior myocardial infarction & $11(30)$ & $7(37)$ & $4(22)$ & 0.476 \\
\hline Coronary artery disease & $27(73)$ & $16(84)$ & $11(61)$ & 0.151 \\
\hline CAG findings & & & & 0.051 \\
\hline 1 vessel disease & $13(48)$ & $5(31)$ & $8(73)$ & $0.034^{*}$ \\
\hline 2 vessel disease & $9(33)$ & $6(38)$ & $3(27)$ & \\
\hline 3 vessel disease & $5(19)$ & $5(31)$ & $0(0)$ & \\
\hline $\mathrm{PCl}$ & $25(68)$ & $16(84)$ & $9(50)$ & 0.038 \\
\hline \multicolumn{5}{|l|}{ Medications } \\
\hline Calcium channel blockers & $16(43)$ & $10(53)$ & $6(33)$ & 0.325 \\
\hline RAS inhibitors & $18(49)$ & $9(47)$ & $9(50)$ & 1.000 \\
\hline Beta-blockers & $22(60)$ & $12(63)$ & $10(56)$ & 0.743 \\
\hline Nitrate & $8(22)$ & $4(21)$ & $4(22)$ & 1.000 \\
\hline Statins & 31 (84) & 18 (95) & $13(72)$ & 0.090 \\
\hline
\end{tabular}

Values are mean \pm standard deviation or number (\%)

*1 vessel vs. 2 - or 3-vessel disease

CAG Coronary angiography, PCI Percutaneous coronary intervention, PWV Pulse wave velocity, RAS Renin-angiotensin system 
Table 3 Comparison of hemodynamic parameters according to the changes of pulse wave velocity after isometric handgrip exercise

\begin{tabular}{|c|c|c|c|}
\hline & $\begin{array}{l}\Delta P W V \geq 0.4 \mathrm{~m} / \mathrm{sec} \\
(N=19)\end{array}$ & $\begin{array}{l}\triangle P W V<0.4 \mathrm{~m} / \mathrm{sec} \\
(N=18)\end{array}$ & $P$ value \\
\hline \multicolumn{4}{|l|}{ Isometric handgrip exercise } \\
\hline Handgrip power, maximal (kg) & $36.2 \pm 13.2$ & $31.0 \pm 11.1$ & 0.207 \\
\hline Handgrip power at exercise (kg) & $13.3 \pm 2.3$ & $11.9 \pm 3.3$ & 0.134 \\
\hline Handgrip power \% & $38.9 \pm 6.6$ & $39.6 \pm 5.8$ & 0.736 \\
\hline \multicolumn{4}{|l|}{ Hemodynamic parameters, baseline } \\
\hline Heart rate (/min) & $62.5 \pm 9.0$ & $66.1 \pm 9.3$ & 0.240 \\
\hline Central systolic BP (mmHg) & $114.3 \pm 18.3$ & $122.7 \pm 22.4$ & 0.216 \\
\hline Central diastolic BP (mmHg) & $60.6 \pm 7.1$ & $66.4 \pm 12.2$ & 0.082 \\
\hline Central pulse pressure $(\mathrm{mmHg})$ & $53.7 \pm 15.6$ & $56.3 \pm 19.8$ & 0.660 \\
\hline Forward pressure $(\mathrm{mmHg})$ & $104.6 \pm 17.3$ & $113.1 \pm 21.1$ & 0.186 \\
\hline Augmentation pressure $(\mathrm{mmHg})$ & $9.7 \pm 7.3$ & $9.6 \pm 5.9$ & 0.974 \\
\hline Augmentation index (\%) & $17.9 \pm 11.9$ & $16.8 \pm 9.2$ & 0.742 \\
\hline Peripheral systolic BP (mmHg) & $122.3 \pm 19.4$ & $127.8 \pm 21.5$ & 0.414 \\
\hline Peripheral diastolic BP (mmHg) & $60.3 \pm 7.7$ & $64.2 \pm 12.0$ & 0.244 \\
\hline Peripheral pulse pressure $(\mathrm{mmHg})$ & $62.0 \pm 17.3$ & $63.6 \pm 19.9$ & 0.787 \\
\hline $\mathrm{PW}(\mathrm{m} / \mathrm{sec})$ & $10.15 \pm 2.07$ & $9.83 \pm 1.61$ & 0.610 \\
\hline \multicolumn{4}{|l|}{ Exercise $3 \mathrm{~min}$} \\
\hline Heart rate (/min) & $68.7 \pm 13.9$ & $72.7 \pm 11.9$ & 0.355 \\
\hline Central systolic BP (mmHg) & $140.7 \pm 20.9$ & $140.1 \pm 25.4$ & 0.935 \\
\hline Central diastolic BP (mmHg) & $70.7 \pm 7.1$ & $74.7 \pm 14.2$ & 0.289 \\
\hline Central pulse pressure $(\mathrm{mmHg})$ & $70.0 \pm 18.0$ & $65.3 \pm 19.5$ & 0.455 \\
\hline Forward pressure (mmHg) & $124.1 \pm 20.2$ & $128.4 \pm 25.3$ & 0.567 \\
\hline Augmentation pressure $(\mathrm{mmHg})$ & $16.6 \pm 9.3$ & $11.7 \pm 7.3$ & 0.081 \\
\hline Augmentation index (\%) & $24.3 \pm 12.4$ & $18.3 \pm 10.3$ & 0.121 \\
\hline Peripheral systolic BP (mmHg) & $144.3 \pm 23.6$ & $136.9 \pm 26.5$ & 0.377 \\
\hline Peripheral diastolic BP (mmHg) & $65.7 \pm 7.7$ & $64.4 \pm 12.7$ & 0.708 \\
\hline Peripheral pulse pressure $(\mathrm{mmHg})$ & $78.6 \pm 21.3$ & $72.6 \pm 21.6$ & 0.395 \\
\hline PWV (m/sec) & $12.03 \pm 2.37$ & $9.51 \pm 1.44$ & $<0.001$ \\
\hline$\Delta$ Central systolic BP $(\mathrm{mmHg})$ & $26.4 \pm 10.3$ & $17.3 \pm 15.6$ & 0.043 \\
\hline$\Delta$ Forward pressure $(\mathrm{mmHg})$ & $19.5 \pm 7.5$ & $15.3 \pm 13.4$ & 0.245 \\
\hline$\Delta$ Augmentation index (\%) & $6.3 \pm 6.3$ & $1.5 \pm 6.8$ & 0.033 \\
\hline$\Delta$ Peripheral systolic BP $(\mathrm{mmHg})$ & $22.1 \pm 11.8$ & $9.1 \pm 8.7$ & 0.001 \\
\hline$\Delta \mathrm{PWV}(\mathrm{m} / \mathrm{sec})$ & $1.89 \pm 1.00$ & $-0.32 \pm 0.49$ & $<0.001$ \\
\hline
\end{tabular}

Values are mean \pm standard deviation

$B P$ Blood pressure, PWV Pulse wave velocity

\section{Discussion}

The main result of this study is that, although a significant correlation was observed between $\triangle \mathrm{PWV}$ and $\triangle \mathrm{AIx}$ after isometric handgrip exercise, only higher $\triangle \mathrm{PWV}$ was associated with the lesion extent of CAD and the necessity for coronary revascularization.

Atherosclerosis is a generalized process of the arterial system including not only coronary arteries but also larger arteries. The atherosclerotic change of the aorta makes the arterial system stiffer, and the stiff aorta is associated with myocardial ischemia and incident CAD [7-9]. Therefore, indexes of arterial stiffness are usually increased in patients with CAD. However, these indexes are often increased in elderly without CAD because systolic BP increases with aging, there is a close association between systolic BP and PWV, and both vascular aging and hypertension are the powerful factors determining arterial stiffness [1]. As a result, the difference of indexes 
Table 4 Comparison of baseline characteristics according to the changes of augmentation index after isometric handgrip exercise

\begin{tabular}{|c|c|c|c|c|}
\hline & $\begin{array}{l}\text { All patients } \\
(N=37)\end{array}$ & $\begin{array}{l}\Delta \mathrm{Alx} \geq 3.3 \% \\
(N=19)\end{array}$ & $\begin{array}{l}\Delta \text { Alx }<3.3 \% \\
(N=18)\end{array}$ & $P$ value \\
\hline Age (years) & $63.3 \pm 9.4$ & $61.7 \pm 10.4$ & $65.0 \pm 8.1$ & 0.288 \\
\hline Male & $23(62)$ & $15(79)$ & $8(44)$ & 0.045 \\
\hline Height (cm) & $160.8 \pm 8.9$ & $162.6 \pm 8.4$ & $158.9 \pm 9.3$ & 0.213 \\
\hline Weight (kg) & $65.7 \pm 12.4$ & $69.2 \pm 13.1$ & $62.1 \pm 10.8$ & 0.081 \\
\hline Body mass index $\left(\mathrm{kg} / \mathrm{m}^{2}\right)$ & $25.27 \pm 3.30$ & $25.98 \pm 3.17$ & $24.51 \pm 3.36$ & 0.180 \\
\hline Smoker & $9(24)$ & $5(26)$ & $4(22)$ & 0.124 \\
\hline Hypertension & $26(70)$ & $13(68)$ & $13(72)$ & 1.000 \\
\hline Diabetes mellitus & $8(22)$ & $3(16)$ & $5(28)$ & 0.447 \\
\hline Hyperlipidemia & $33(89)$ & $18(95)$ & $15(83)$ & 0.340 \\
\hline Chronic kidney disease & $19(51)$ & $8(42)$ & $11(61)$ & 0.330 \\
\hline Prior myocardial infarction & $11(30)$ & $5(26)$ & $6(33)$ & 0.728 \\
\hline Coronary artery disease & $27(73)$ & $15(79)$ & $12(67)$ & 0.476 \\
\hline CAG findings & & & & 0.620 \\
\hline 1 vessel disease & $13(48)$ & $6(40)$ & $7(58)$ & $0.313^{*}$ \\
\hline 2 vessel disease & $9(33)$ & $6(40)$ & $3(25)$ & \\
\hline 3 vessel disease & $5(19)$ & $3(20)$ & $2(17)$ & \\
\hline $\mathrm{PCl}$ & $25(68)$ & $13(68)$ & $12(67)$ & 1.000 \\
\hline \multicolumn{5}{|l|}{ Medications } \\
\hline Calcium channel blockers & $16(43)$ & $10(53)$ & $6(33)$ & 0.325 \\
\hline RAS inhibitors & $18(49)$ & $9(47)$ & $9(50)$ & 1.000 \\
\hline Beta-blockers & $22(60)$ & $13(68)$ & $9(50)$ & 0.325 \\
\hline Nitrate & $8(22)$ & $4(21)$ & $4(22)$ & 1.000 \\
\hline Statins & $31(84)$ & $18(95)$ & $13(72)$ & 0.090 \\
\hline
\end{tabular}

Values are mean \pm standard deviation or number (\%)

*1 vessel vs. 2- or 3-vessel disease

Alx Augmentation index, CAG Coronary angiography, PCI Percutaneous coronary intervention, RAS Renin-angiotensin system

of arterial stiffness between patients with and without CAD may be not evident in elderly. The previous metaanalysis showed that aortic PWV was a predictor of future cardiovascular events such as CAD or stroke even in elderly, but its relation to cardiovascular events was weaker in older than younger patients, and the hazard ratio was decreased with age $(1.89,1.77,1.36$, and 1.23 for age $\leq 50,51-60,61-70$, and $>70$ years, respectively) [9]. The usefulness of AIx as a marker for CAD in older patients is even more unclear. Because AIx strongly correlates with age and it is also influenced by heart rate, body length or the pattern of ventricular ejection [3], AIx may not be an accurate index for arterial stiffness. The comparison of aortic PWV with AIx as a surrogate for the extent and severity of CAD was possible in one study that measured both indexes in the same patients at the time of coronary angiography. It showed the significant association of the extent and severity of CAD with PWV, but not with AIx [12].

Isometric handgrip exercise is a non-invasive and easily applicable maneuver to increase heart rate, BP and left ventricular afterload [21-23]. In this study, central systolic BP, forward and augmentation pressures, AIx, peripheral $\mathrm{BP}$ and PWV were increased after handgrip exercise. Although $\triangle \mathrm{PWV}$ and $\triangle \mathrm{AIx}$ correlated each other, the pattern of $\triangle \mathrm{PWV}$ and $\triangle \mathrm{AIx}$ after handgrip exercise was different. Baseline PWV was not different between higher and lower $\triangle \mathrm{PWV}$ groups, but after 3-min handgrip exercise, $\Delta$ central systolic $B P, \Delta$ peripheral systolic $\mathrm{BP}$ and $\mathrm{PWV}$ were greater in higher $\triangle \mathrm{PWV}$ group. However, baseline AIx was smaller in higher $\triangle \mathrm{AIx}$ group, and AIx after 3-min handgrip exercise became similar between higher and lower $\Delta$ AIx groups because of more increase of augmentation pressure in lower $\Delta$ AIx group. All of these findings suggest that $\Delta$ AIx may be larger in patients with lower baseline AIx regardless of arterial stiffness and reach plateau after handgrip exercise. Therefore, $\Delta$ AIx after handgrip exercise may not be as a good index for arterial stiffness as $\triangle \mathrm{PWV}$.

The mean age of patients of this study was 63.3 years, $65 \%$ were $>60$ years and $70 \%$ of them had hypertension. These findings may be usually seen in patients with 
Table 5 Comparison of hemodynamic parameters according to the changes of augmentation index after isometric handgrip exercise

\begin{tabular}{|c|c|c|c|}
\hline & $\begin{array}{l}\Delta \mathrm{Alx} \geq 3.3 \% \\
(N=19)\end{array}$ & $\begin{array}{l}\Delta \text { Alx }<3.3 \% \\
(N=18)\end{array}$ & $P$ value \\
\hline \multicolumn{4}{|l|}{ Isometric handgrip exercise } \\
\hline Handgrip power, maximal (kg) & $37.0 \pm 14.4$ & $30.1 \pm 8.7$ & 0.089 \\
\hline Handgrip power at exercise (kg) & $13.5 \pm 2.8$ & $11.7 \pm 2.7$ & 0.060 \\
\hline Handgrip power \% & $38.7 \pm 6.7$ & $39.8 \pm 5.6$ & 0.566 \\
\hline \multicolumn{4}{|l|}{ Hemodynamic parameters, baseline } \\
\hline Heart rate (/min) & $65.7 \pm 9.9$ & $62.7 \pm 8.4$ & 0.334 \\
\hline Central systolic BP (mmHg) & $109.9 \pm 16.2$ & $127.3 \pm 21.3$ & 0.008 \\
\hline Central diastolic BP (mmHg) & $62.0 \pm 8.4$ & $65.0 \pm 12.0$ & 0.373 \\
\hline Central pulse pressure $(\mathrm{mmHg})$ & $48.0 \pm 14.7$ & $62.3 \pm 17.7$ & 0.011 \\
\hline Forward pressure $(\mathrm{mmHg})$ & $103.7 \pm 15.8$ & $114.1 \pm 21.9$ & 0.105 \\
\hline Augmentation pressure $(\mathrm{mmHg})$ & $6.2 \pm 4.9$ & $13.3 \pm 6.2$ & $<0.001$ \\
\hline Augmentation index (\%) & $13.3 \pm 9.6$ & $21.7 \pm 10.0$ & 0.013 \\
\hline Peripheral systolic BP (mmHg) & $118.5 \pm 19.3$ & $131.8 \pm 19.8$ & 0.045 \\
\hline Peripheral diastolic BP (mmHg) & $61.3 \pm 8.3$ & $63.2 \pm 11.9$ & 0.562 \\
\hline Peripheral pulse pressure $(\mathrm{mmHg})$ & $57.2 \pm 17.8$ & $68.6 \pm 17.6$ & 0.058 \\
\hline Pulse wave velocity (m/sec) & $9.30 \pm 1.72$ & $10.72 \pm 1.72$ & 0.017 \\
\hline \multicolumn{4}{|l|}{ Exercise $3 \mathrm{~min}$} \\
\hline Heart rate (/min) & $70.5 \pm 13.4$ & $70.8 \pm 12.9$ & 0.948 \\
\hline Central systolic BP (mmHg) & $135.0 \pm 19.7$ & $146.1 \pm 25.1$ & 0.140 \\
\hline Central diastolic BP (mmHg) & $71.5 \pm 7.2$ & $73.9 \pm 14.4$ & 0.527 \\
\hline Central pulse pressure $(\mathrm{mmHg})$ & $63.5 \pm 18.7$ & $72.2 \pm 18.0$ & 0.157 \\
\hline Forward pressure (mmHg) & $120.9 \pm 17.0$ & $131.7 \pm 26.7$ & 0.154 \\
\hline Augmentation pressure $(\mathrm{mmHg})$ & $14.1 \pm 9.1$ & $14.4 \pm 8.5$ & 0.908 \\
\hline Augmentation index (\%) & $22.2 \pm 11.6$ & $20.4 \pm 12.0$ & 0.648 \\
\hline Peripheral systolic BP (mmHg) & $136.2 \pm 22.6$ & $145.6 \pm 27.1$ & 0.259 \\
\hline Peripheral diastolic BP (mmHg) & $64.5 \pm 7.6$ & $65.6 \pm 12.8$ & 0.754 \\
\hline Peripheral pulse pressure $(\mathrm{mmHg})$ & $71.6 \pm 21.1$ & $79.9 \pm 21.4$ & 0.242 \\
\hline Pulse wave velocity (m/sec) & $10.68 \pm 2.59$ & $10.94 \pm 2.10$ & 0.747 \\
\hline$\Delta$ Central systolic BP $(\mathrm{mmHg})$ & $25.1 \pm 11.7$ & $18.8 \pm 15.3$ & 0.169 \\
\hline$\Delta$ Forward pressure $(\mathrm{mmHg})$ & $17.2 \pm 7.8$ & $17.7 \pm 13.6$ & 0.900 \\
\hline$\Delta$ Augmentation index (\%) & $9.0 \pm 5.3$ & $-1.3 \pm 3.8$ & $<0.001$ \\
\hline$\Delta$ Peripheral systolic BP (mmHg) & $17.7 \pm 11.2$ & $13.7 \pm 13.1$ & 0.330 \\
\hline$\Delta$ Pulse wave velocity (m/sec) & $1.38 \pm 1.39$ & $0.21 \pm 1.08$ & 0.008 \\
\hline
\end{tabular}

Values are mean \pm standard

Alx Augmentation index, BP Blood pressure

CAD. In this clinical setting, simple PWV value may not differentiate patients with CAD and the lesion severity of CAD. In the previous study, we showed that PWV value at rest was not different between patients with and without CAD, but after 3-min handgrip exercise, PWV was significantly increased only in patients with CAD [24]. However, the change of PWV after handgrip exercise showed a diverse pattern such that PWV increased even in patients without CAD, and a cut-off point of $\triangle \mathrm{PWV}$ after handgrip exercise, which was associated with the lesion extent of CAD and the necessity for coronary revascularization, was not evaluated in the previous study. In this study, higher $\triangle \mathrm{PWV}$ group had more 2- or 3-vessel CAD and underwent more PCI than lower $\triangle \mathrm{PWV}$ group. On the other hand, the lesion extent of $\mathrm{CAD}$ and the necessity for PCI were not different between higher and lower $\triangle \mathrm{AIx}$ groups. In addition, Cstatistics and multiple logistic regression analysis showed 


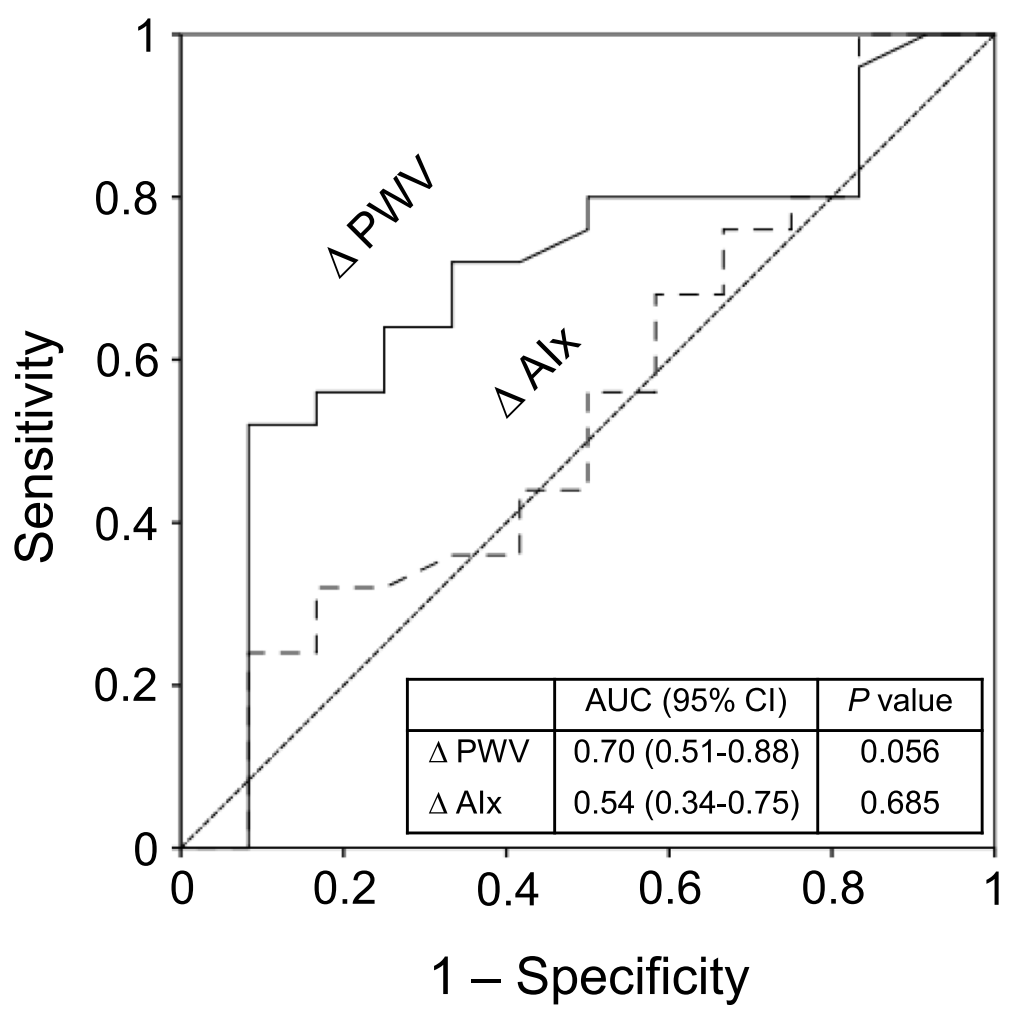

Fig. 3 Area under curve (AUC) with 95\% confidence interval (Cl) of $\Delta$ pulse wave velocity (PWV) or $\Delta$ augmentation index (Alx) in association with percutaneous coronary intervention by $\mathrm{C}$-statistics

that $\triangle \mathrm{PWV}$ was associated with $\mathrm{PCI}$ but not $\triangle \mathrm{AIx}$. These findings suggest that $\triangle \mathrm{PWV}$ may be used as an index for differentiating the lesion extent of CAD and the necessity for coronary revascularization even in elderly patients with hypertension, but $\Delta$ AIx may not.

This study has several limitations. First, a fluid-filled catheter system, instead of a high-fidelity micromanometer, was used to record the pressure waveforms. A definite merging point of the forward and the reflected waves on the recorded pressure waveform was not identified in aortic pressure waveforms of 4 patients (9.5\%). Second, over half of patients were taking medications with a vasodilation property, which may influence the change of BP, PWV, and AIx after handgrip exercise. However such medications were equally taken in both higher and lower $\triangle \mathrm{PWV}$ or $\triangle \mathrm{AIx}$ groups, and baseline central and peripheral BP were not different between groups. Third, PCI performed not only on the day of the study but also 1 year ago was counted as having coronary revascularization. Including only prospectively conducted coronary revascularization would have increased the feasibility of the study. Fourth, the number of patients might not be enough to accurately evaluate the changes of PWV and AIx after handgrip exercise. Nevertheless, the association between higher $\triangle \mathrm{PWV}$ and the lesion extent of CAD or the necessity for PCI was demonstrated.

This was an invasive study that measured hemodynamic parameters during CAG. To be widely use, the applicability of a non-invasive method measuring $\triangle \mathrm{PWV}$ or $\triangle \mathrm{AIx}$ after handgrip exercise using an applanation tonometry needs to be validated in a future study.

\section{Conclusions}

Both PWV and AIx increased after isometric handgrip exercise, and $\triangle \mathrm{PWV}$ was significantly correlated with $\triangle \mathrm{AIx}$. However, higher $\triangle \mathrm{PWV}$ was associated with the lesion extent of coronary atherosclerosis and the necessity for coronary revascularization, but higher $\Delta$ AIx was not.

\section{Abbreviations}

Alx: Augmentation index; AP: Augmentation pressure; CAD: Coronary artery disease; CAG: Coronary angiography; PCl: Percutaneous coronary intervention; PWV: Pulse wave velocity; RAS: Renin-angiotensin system

\section{Acknowledgements}

This work was supported by the 2021 education, research and student guidance grant funded by Jeju National University. 


\section{Authors' contributions}

SK and SJ conceived of this study, participated in its design and coordination, analyzing data, and writing a draft of this manuscript. YK, JB, and KB participated in obtaining written informed consents from patients, obtaining waveforms, and data analysis. JL, JC, and SK, participated in data analysis, and helped to conduct the literature review and draft the manuscript. All authors read and approved the final manuscript.

\section{Funding}

This work was supported by the 2021 education, research and student guidance grant funded by Jeju National University.

\section{Availability of data and materials}

The datasets used and/or analyzed during the current study are available from the corresponding author on reasonable request.

\section{Ethics approval and consent to participate}

The study protocol was approved by the institutional review board of Jeju National University Hospital (IRB 2011-19). Written informed consents were obtained from participating patients or legal representative.

\section{Consent for publication}

Not applicable.

\section{Competing interests}

The authors declare that they have no competing interests.

\section{Author details}

'Department of Internal Medicine, Jeju National University Hospital, 15 Aran 13-gil, Jeju City, Jeju Province 63241, Republic of Korea. ${ }^{2}$ Department of Internal Medicine, Jeju National University School of Medicine, 15 Aran 13-gil, Jeju City, Jeju Province 63241, Republic of Korea. ${ }^{3}$ Institute of Medical Science, Jeju National University, 15 Aran 13-gil, Jeju City, Jeju Province 63241, Republic of Korea.

Received: 14 October 2020 Accepted: 13 January 2021

Published online: 01 May 2021

\section{References}

1. Cavalcante JL, Lima JA, Redheuil A, Al-Mallah MH. Aortic stiffness: current understanding and future directions. J Am Coll Cardiol. 2011;57:1511-22.

2. Lee JG, Joo SJ. Arterial stiffness and cardiovascular risk. Korean J Intern Med. 2019;34:504-6

3. Nichols WW, O'Rourke MF, Vlachopoulos C. Wave reflection. In: McDonald's blood flow in arteries. 6th ed. Boca Raton: CRC Press; 2011. p. 195-223.

4. Kingwell BA, Waddell TK, Medley TL, Cameron JD, Dart AM. Large artery stiffness predicts ischemic threshold in patients with coronary artery disease. J Am Coll Cardiol. 2002:40:773-9.

5. Ikonomidis I, Makavos G, Lekakis J. Arterial stiffness and coronary artery disease. Curr Opin Cardiol. 2015;30:422-31.

6. Vlachopoulos C, Xaplanteris P, Aboyans V, Brodmann M, Cífková R Cosentino F, De Carlo M, Gallino A, Landmesser U, Laurent S, Lekakis J, Mikhailidis DP, Naka KK, Protogerou AD, Rizzoni D, Schmidt-Trucksäss A, Van Bortel L, Weber T, Yamashina A, Zimlichman R, Boutouyrie P, Cockcroft J, O'Rourke M, Park JB, Schillaci G, Sillesen H, Townsend RR. The role of vascular biomarkers for primary and secondary prevention. A position paper from the European Society of Cardiology Working Group on peripheral circulation: endorsed by the Association for Research into arterial structure and physiology (ARTERY) society. Atherosclerosis. 2015;241:507-32.

7. Mattace-Raso FU, van der Cammen TJ, Hofman A, van Popele NM, Bos ML, Schalekamp MA, Asmar R, Reneman RS, Hoeks AP, Breteler MM, Witteman $J$ J. Arterial stiffness and risk of coronary heart disease and stroke: the Rotterdam study. Circulation. 2006;113:657-63.

8. Mitchell GF, Hwang SJ, Vasan RS, Larson MG, Pencina MJ, Hamburg NM, Vita JA, Levy D, Benjamin EJ. Arterial stiffness and cardiovascular events: the Framingham heart study. Circulation. 2010;121:505-11.

9. Ben-Shlomo Y, Spears M, Boustred C, May M, Anderson SG, Benjamin EJ, Boutouyrie P, Cameron J, Chen CH, Cruickshank JK, Hwang SJ, Lakatta EG, Laurent S, Maldonado J, Mitchell GF, Najjar SS, Newman AB, Ohishi M, Pannier B, Pereira T, Vasan RS, Shokawa T, Sutton-Tyrell K, Verbeke F, Wang KL, Webb DJ, Willum Hansen T, Zoungas S, McEniery CM, Cockcroft JR,
Wilkinson IB. Aortic pulse wave velocity improves cardiovascular event prediction: an individual participant meta-analysis of prospective observational data from 17,635 subjects. J Am Coll Cardiol. 2014;63:636-46.

10. Covic A, Haydar AA, Bhamra-Ariza P, Gusbeth-Tatomir P, Goldsmith DJ. Aortic pulse wave velocity and arterial wave reflections predict the extent and severity of coronary artery disease in chronic kidney disease patients. J Nephrol. 2005;18:388-96.

11. Kullo IJ, Bielak LF, Turner ST, Sheedy PF 2nd, Peyser PA. Aortic pulse wave velocity is associated with the presence and quantity of coronary artery calcium: a community-based study. Hypertension. 2006;47:174-9.

12. Hope SA, Antonis P, Adam D, Cameron JD, Meredith IT. Arterial pulse wave velocity but not augmentation index is associated with coronary artery disease extent and severity: implications for arterial transfer function applicability. J Hypertens. 2007;25:2105-9.

13. Bechlioulis A, Vakalis K, Naka KK, Bourantas CV, Papamichael ND, Kotsia A, Tzimas T, Pappas K, Katsouras CS, Michalis LK. Increased aortic pulse wave velocity is associated with the presence of angiographic coronary artery disease in overweight and obese patients. Am J Hypertens. 2013;26:265-70.

14. Kim HL, Kim SH. Pulse wave velocity in atherosclerosis. Front Cardiovasc Med. 2019;6:41.

15. Williams B, Mancia G, Spiering W, Agabiti Rosei E, Azizi M, Burnier M, Clement DL, Coca A, de Simone G, Dominiczak A, Kahan T, Mahfoud F, Redon J, Ruilope L, Zanchetti A, Kerins M, Kjeldsen SE, Kreutz R, Laurent S, Lip GYH, McManus R, Narkiewicz K, Ruschitzka F, Schmieder RE, Shlyakhto E, Tsioufis C, Aboyans V, Desormais I. Authors/task force members:. 2018 ESC/ ESH guidelines for the management of arterial hypertension: the task force for the management of arterial hypertension of the European Society of Cardiology and the European Society of Hypertension: the task force for the management of arterial hypertension of the European Society of Cardiology and the European Society of Hypertension. J Hypertens. 2018;36:1953-2041.

16. Lee HY, Shin J, Kim GH, Park S, Ihm SH, Kim HC, Kim Kl, Kim JH, Lee JH, Park JM, Pyun WB, Chae SC. 2018 Korean Society of Hypertension Guidelines for the management of hypertension: part II-diagnosis and treatment of hypertension. Clin Hypertens. 2019;25:20.

17. Weber T, Auer J, O'Rourke MF, Kvas E, Lassnig E, Berent R, Eber B. Arterial stiffness, wave reflections, and the risk of coronary artery disease. Circulation. 2004:109:184-9.

18. Cho SW, Kim BK, Kim JH, Byun YS, Goh CW, Rhee KJ, Ahn HS, Lee BK, Kim BO. Non-invasively measured aortic wave reflection and pulse pressure amplification are related to the severity of coronary artery disease. J Cardiol. 2013;62:131-7.

19. Choi J, Kim SY, Joo SJ, Kim KS. Augmentation index is associated with coronary revascularization in patients with high Framingham risk scores: a hospital-based observational study. BMC Cardiovasc Disord. 2015;15:131.

20. Hayashi S, Yamada H, Bando M, Hotchi J, Ise T, Yamaguchi K, Iwase T, Soeki T, Wakatsuki T, Tamaki T, Sata M. Augmentation index does not reflect risk of coronary artery disease in elderly patients. Circ J. 2014;78:1176-82.

21. Kivowitz C, Parmley WW, Donoso R, Marcus H, Ganz W, Swan HJ. Effects of isometric exercise on cardiac performance. The grip test. Circulation. 1971; 44:994-1002.

22. Fisher ML, Nutter DO, Jacobs W, Schlant RC. Haemodynamic responses to isometric exercise (handgrip) in patients with heart disease. Br Heart J. 1973; 35:422-32.

23. Martin CE, Shaver JA, Leon DF, Thompson ME, Reddy PS, Leonard JJ. Autonomic mechanisms in hemodynamic responses to isometric exercise. $J$ Clin Invest. 1974;54:104-15

24. Moon SH, Moon JC, Heo DH, Lim YH, Choi JH, Kim SY, Kim KS, Joo SJ. Increased pulse wave velocity and augmentation index after isometric handgrip exercise in patients with coronary artery disease. Clin Hypertens. 2015;21:5.

\section{Publisher's Note}

Springer Nature remains neutral with regard to jurisdictional claims in published maps and institutional affiliations. 